Gefässchirurgie 2021 26:635-638 https://doi.org/10.1007/s00772-021-00829-2

Angenommen: 15. September 2021

Online publiziert: 2. November 2021

๑) Der/die Autor(en) 2021

\section{Spinaler Querschnitt nach EVAR bei bestehendem Nierenzellkarzinom}

\author{
Johanna Kuchar' · Björn Sommer ${ }^{2}$ Ehab Shiban² $\cdot$ Yvonne N. Gosslau' . \\ Alexander Hyhlik-Dürr' \\ ' Gefäßchirurgie und endovaskuläre Chirurgie, Medizinische Fakultät, Universität Augsburg, Augsburg, \\ Deutschland \\ ${ }^{2}$ Klinik für Neurochirurgie, Medizinische Fakultät, Universität Augsburg, Augsburg, Deutschland
}

Zum Auftreten einer spinalen Ischämie nach endovaskulärer Versorgung von Aneurysmata gibt es in der Literatur schwankende Angaben zwischen 0,21\% [1] und $17,7 \%$ [2]. Pathophysiologisch liegt eine von zuführenden arteriellen Ästen entstehende verminderte Durchblutung des ventralen Rückenmarks zugrunde. Das Risiko steigt mit der Anzahl der überstenteten Lumbalarterien.

Zur Therapie eines A.-spinalis-anteriorSyndroms erfolgt die Blutdruckanhebung, die Anlage eines spinalen Katheters und die intensivmedizinische Überwachung. In einigen Fällen führt dies zur Regredienz der Symptomatik [3].

Wir berichten über einen Fall einer Paraparese nach endovascular aortic repair (EVAR) der infrarenalen Aorta und deren mögliche Differentialdiagnosen zum A.spinalis-anterior-Syndrom.

\section{Anamnese}

Es erfolgt die Übernahme eines 84-jährigen multimorbiden Patienten (art., pulmonale Hypertonie, Vorhofflimmern [VHF], Schrittmacher [SM], Niereninsuffizienz) aus einem externen Haus aufgrund abdomineller Schmerzen bei seit Jahren bekanntem infrarenalen Aortenaneurysma (AA). Bisher wünschte der Patient keine Versorgung des bestehenden Aneurysmas.

\section{Befund und Diagnose}

Stationäre Aufnahme des Patienten zur Blutdruckeinstellung, international nor- malized ratio (INR)-Normalisierung bei Entgleisung und kardialen Abklärung. Zur weiteren Diagnostik erfolgt eine Sonographie des Abdomens, eine Computertomographie-Angiographie (CT-A) zur Darstellung des Aneurysmas und zum Ausschluss weiterer Pathologien.

In der Sonographie und CT-A zeigt sich ein infrarenales AA mit 7,7 cm Durchmesser (- Abb. 1).

Nebenbefundlich zeigt sich eine Kontrastmittel aufnehmende RF der linken Niere ca. 4,6 cm Durchmesser und hoch suspekt auf ein Nierenzellkarzinom. Zudem zeigt sich eine osteolytische RF am linken oberen Schambeinast, passend zu einer ossären Metastase.

Der Patient wünscht nun ausdrücklich die operative Versorgung des AA bei fortbestehender abdomineller Symptomatik und Rupturgefahr. Präoperativ erfolgt die urologische Vorstellung und ergänzend ein Staging-CT-Thorax. Eine pulmonale Raumforderung wird ausgeschlossen. Weitere Metastasen werden nicht detektiert.

Die weitere Tumorabklärung ist aufgrund der dringlichen Versorgungsindikation für den postoperativen Zeitraum geplant.

\section{Therapie und Verlauf}

Es erfolgt die endovaskuläre Versorgung des Patienten mittels Standardprothese: EVAR, TREO 26/14/100, Re: 15/15/140, li: $15 / 15 / 140$. Bei ausgeprägter Verkalkung erfolgt zudem eine perkutane transluminale Angioplastie (PTA) der Arteria Iliaca communis (AIC) und der Arteria Ilia- 


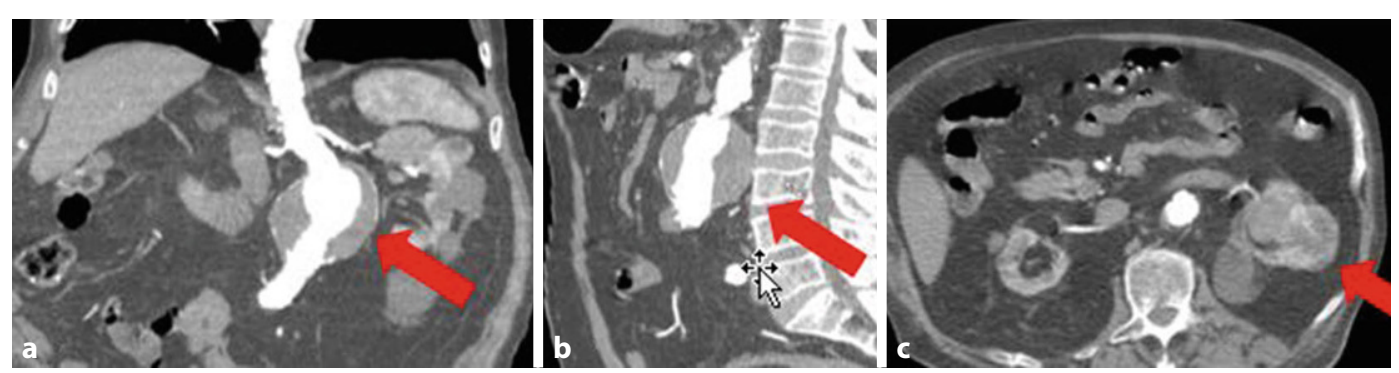

Abb. $1 \Delta$ CT-A der Aorta: infrarenales Aortenaneurysma mit 7,7 cm Durchmesser und randständiger Thrombosierung. a Koronares Schnittbild (Aneurysma s. Pfeil), b sagittales Schnittbild (Aneurysma s. Pfeil), c Kontrastmittel aufnehmende Raumforderung (RF) in der linke Niere (s. Pfeil)
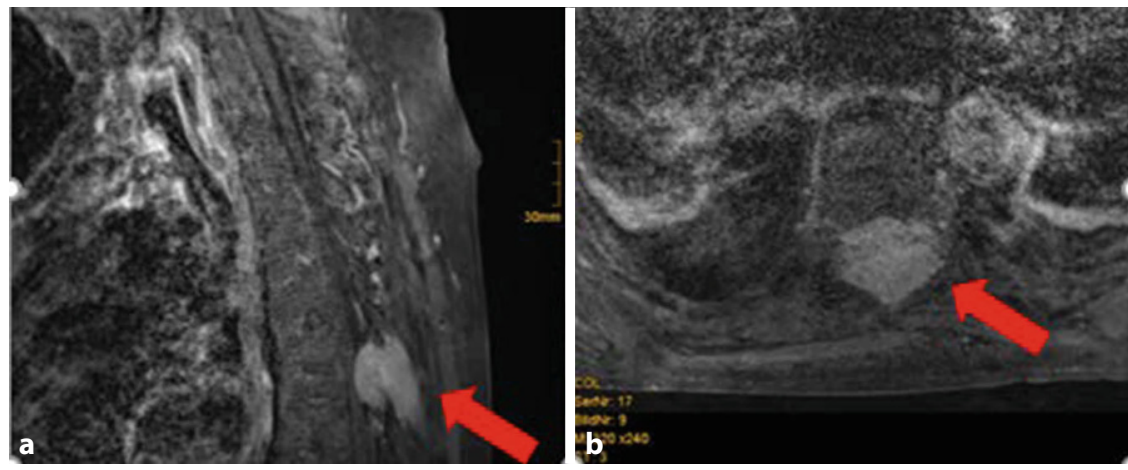

Abb. 2 ム Kontrastmittelverstärktes MRT der Wirbelsäule mit hochgradiger Myelonkompression in Höhe BW6

Neurologisches Defizit n. EVAR
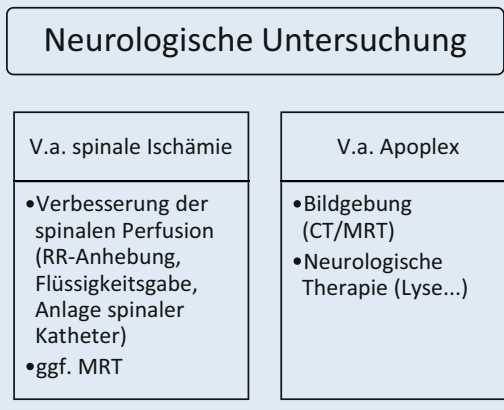

Abklärung Durchblutungssituation (Pulse, Doppler, Sonographie)

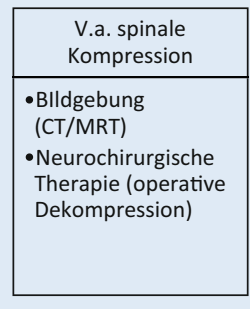

Im weiteren Verlauf keine Besserung der Symptomatik, sodass trotz SM ein MRT der Wirbelsäule erfolgt. Hier zeigt sich eine ausgedehnte osteolytische Metastase in den dorsalen Anhangsgebilden des BWK 6 mit Myelonkompression. (• Abb. 2). Eine spinale Ischämie zeigt sich nicht.

Es besteht die Indikation zur sofortigen dorsalen Tumordekompression. Bereits während der Narkoseeinleitung ist der Patient kreislaufinstabil und benötigte eine hoch dosierte medikamentöse Kreislaufunterstützung. Nach Tumorlaminektomie musste das Tumordebulking aufgrund der instabilen Hämodynamik bei starken Blutungen mehrfach unterbrochen werden. Der Patient bot schließlich eine Asystolie, was zu einem Abbruch der Operation und einer Reanimation führte.

Der Patient wurde stabilisiert und auf die Intensivstation verbracht. Dort Festlegung eines palliativen Therapieregimes. Der Patient verstarb im Verlauf.

\section{Diskussion}

Die weitere Tumorabklärung mittels Skelettszintigraphie im Vorfeld der Operation hätte die bis dahin nicht detektierte Metastase auf Höhe von BWK 6 ggf. darstellen können. Zudem ist in der Nachschau bei genauer Auswertung der CT-Bildgebung die Metastase bereits im CT sichtbar, wurde jedoch nicht beschrieben. Eine weitere langwierige Abklärung bei fehlender Symptomatik bzgl. des Nierenzellkarzinoms bzw. der bestehenden WS-Metastase wäre bei dringlicher OP-Indikation nicht infrage gekommen. Das Therapieregime wäre wohl auch bei Vorliegen der Skelettszintigraphie nicht geändert worden, da keinerlei neurologische Symptomatik vorlag. Allerdings wäre postopera- 
Tab. 1 Differentialdiagnosen mit typischer Klinik

\begin{tabular}{|l|l|l|l|l|l|l|}
\hline Diagnose & Motorik & $\begin{array}{l}\text { Epikritische } \\
\text { Sensibilität }\end{array}$ & $\begin{array}{l}\text { Protopathische } \\
\text { Sensibilität }\end{array}$ & $\begin{array}{l}\text { Puls- } \\
\text { status }\end{array}$ & $\begin{array}{l}\text { Diagnos- } \\
\text { tik }\end{array}$ & Mögliche Symptomatik \\
\hline $\begin{array}{l}\text { Arteria-spina- } \\
\text { lis-anterior- } \\
\text { Syndrom }\end{array}$ & - & + & - & + & MRT & $\begin{array}{l}\text { Schlaffe Parese, gürtelförmige Schmerzen/Parästhesie, Be- } \\
\text { einträchtigung von Schmerz- und Temperaturempfinden } \\
\text { bds. bei erhaltenem Berührungs- und Vibrationsempfinden } \\
\text { Blasen/Mastdarmstörung/Impotenz }\end{array}$ \\
\hline Apoplex & $+/-$ & $+/-$ & $+/-$ & + & CT/MRT & $\begin{array}{l}\text { Einseitige Paresen/Sensibilitätsstörungen, Sprachstörun- } \\
\text { gen, Visusstörungen }\end{array}$ \\
\hline $\begin{array}{l}\text { Rückenmarks- } \\
\text { kompression }\end{array}$ & $+/-$ & $+/-$ & $+/-$ & + & CT & $\begin{array}{l}\text { Beidseitige Parese, Parästhesie } \\
\text { Blasen/Mastdarmstörung }\end{array}$ \\
\hline Akute Ischämie & $+/-$ & $+/-$ & $+/-$ & - & CT & Beidseits Schmerzen, blasse und kalte untere Extremität \\
\hline
\end{tabular}

tiv sicherlich nicht zunächst eine spinale Ischämie in Erwägung gezogen worden, sondern die tumorbedingte Querschnittssymptomatik hätte früher erkannt werden können. Zusätzlich ist zu beachten, dass bei spinaler Ischämie typischerweise Motorikstörungen sowie Störungen der protopathischen Sensibilität auftreten, jedoch kein Fehlen der epikritischen Sensibilität wie in diesem Fall ([4]; - Tab. 1).

Da anhand der vorliegenden Bildgebung und der direkt postoperativ aufgetretenen Klinik zunächst von einer spinalen Ischämie ausgegangen wurde und eine MR-Untersuchung durch den SM nur verzögert stattfinden konnte, wurde die komplette Spinalkanalstenose erst spät diagnostiziert. Da bei Knochenmetastasen von Nierenzellkarzinomen häufig eine ausgeprägte Vaskularisation vorliegt, hätte man zur Reduktion des Blutungsrisikos eine präoperative Embolisation der Metastase erwägen können [5]. Die Embolisation verringert dabei nicht nur den Blutverlust, sondern verkürzt auch die OP-Dauer [5]. Es ist zu beachten, dass durch die Embolisation auch ein A.-spinalis-anterior-Syndrom verursacht werden kann [5]. Zudem ist eine vollständige Embolisation nicht immer möglich [5]. Insgesamt stellt die Embolisation jedoch ein sicheres Verfahren zur Reduktion des intraoperativen Blutverlustes dar [5].

Metastasen von Nierenzellkarzinomen weisen in 60-70\% eine Hypervaskularisierung auf, wodurch ein erhöhtes perioperatives Blutungsrisiko sowie postoperatives Rezidivblutungsrisiko vorliegt [5]. In Abhängigkeit von Komorbiditäten, ASAScore und Ausprägung der Metastasierung weisen Patienten wie in dem vorgestellten Fall selbst ohne Operation hohe Mortalitätsraten auf. Sollte eine spi- nale Tumoraussaat zur Kompression des Rückenmarks mit neurologischen Defiziten führen, wird je nach Lokalisation und Stabilität eine Tumordekompression empfohlen. Eine präoperative Embolisation von Nierenzelltumormetastasen soll erwogen werden. Bezüglich des klinisch relevanten Nutzens existiert bislang keine eindeutige evidenzbasierte Empfehlung [5]. Auch sind periprozedurale schwerwiegende Komplikationen der endovaskulären Intervention wie zerebrale/spinale Ischämie oder ödematöse Schwellung des intraspinalen Tumorgewebes durch die Embolisatpartikel zu berücksichtigen, die jedoch selten auftreten. Ein eindeutiges Zeitintervall zwischen Embolisation und dem chirurgischen Eingriff besteht nicht, generell wird eine Operation innerhalb von $48 \mathrm{~h}$ angestrebt [5]. Im vorliegenden Fall wurde eine Embolisation in Anbetracht der Schwere des neurologischen Defizites mit rapider Verschlechterung nicht durchgeführt.

Ein spezifischer diagnostischer Algorithmus sollte bei neurologischem Defizit nach EVAR vorliegen (- Abb. 3).

\section{Fazit für die Praxis}

- Bei großen Karzinomen mit Neigung zu ossärer Metastasierung sollte differentialdiagnostisch auch an eine metastasenbedingte Myelonkompression gedacht werden.

- Da Knochenmetastasen von Nierenzellkarzinomen häufig gut vaskularisiert sind, sollte eine präoperative Embolisation erwogen werden.

- Eine genaue neurologische Untersuchung mit Beachtung der Sensibilitätsqualität ist für eine frühe Diagnosestellung essenziell.

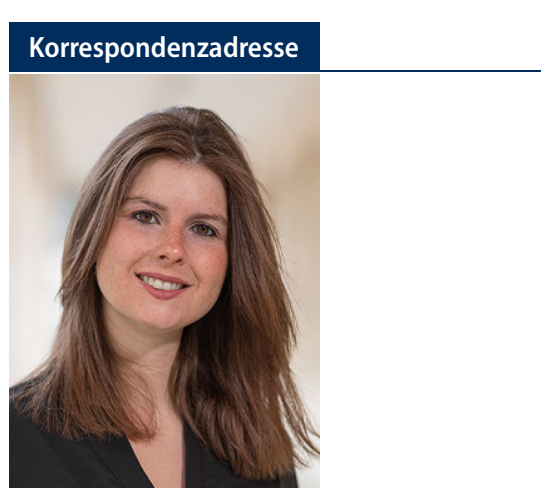

Johanna Kuchar

Gefäßchirurgie und endovaskuläre Chirurgie, Medizinische Fakultät, Universität Augsburg Stenglinstr. 2, 86156 Augsburg, Deutschland johanna.kuchar@uk-augsburg.de

Funding. Open Access funding enabled and organized by Projekt DEAL.

\section{Einhaltung ethischer Richtlinien}

Interessenkonflikt. J. Kuchar, B. Sommer, E. Shiban, Y.N. Gosslau und A. Hyhlik-Dürr geben an, dass kein Interessenkonflikt besteht.

Für diesen Beitrag wurden von den Autoren keine Studien an Menschen oder Tieren durchgeführt. Für die aufgeführten Studien gelten die jeweils dort angegebenen ethischen Richtlinien. Für Bildmaterial oder anderweitige Angaben innerhalb des Manuskripts, über die Patienten zu identifizieren sind, liegt von innen und/oder ihren gesetzlichen Vertretern eine schriftliche Einwilligung vor.

Open Access. Dieser Artikel wird unter der Creative Commons Namensnennung 4.0 International Lizenz veröffentlicht, welche die Nutzung, Vervielfältigung, Bearbeitung, Verbreitung und Wiedergabe in jeglichem Medium und Format erlaubt, sofern Sie den/die ursprünglichen Autor(en) und die Quelle ordnungsgemäß nennen, einen Link zur Creative Commons Lizenz beifügen und angeben, ob Änderungen vorgenommen wurden.

Die in diesem Artikel enthaltenen Bilder und sonstiges Drittmaterial unterliegen ebenfalls der genannten 


\section{Der interessante Fall}

Creative Commons Lizenz, sofern sich aus der Abbildungslegende nichts anderes ergibt. Sofern das betreffende Material nicht unter der genannten Creative Commons Lizenz steht und die betreffende Handlung nicht nach gesetzlichen Vorschriften erlaubt ist, ist für die oben aufgeführten Weiterverwendungen des Materials die Einwilligung des jeweiligen Rechteinhabers einzuholen.

Weitere Details zur Lizenz entnehmen Sie bitte der Lizenzinformation auf http://creativecommons.org/ licenses/by/4.0/deed.de.

\section{Literatur}

1. Berg P, Kaufmann D, van MarrewijkCJ, Buth J(2001) Spinal cord ischaemia after stent-graft treatment for infra-renal abdominal aortic aneurysms. Analysis of the Eurostar database. Eur J Vasc Endovasc Surg 22:342-347. https://doi.org/10. 1053/ejvs.2001.1470

2. Spanos K, Kölbel T, Kubitz JC, Wipper S, Konstantinou N, Heidemann F, Rohlffs F, Debus SE, Tsilimparis N (2018) Risk of spinal cord ischemia after fenestrated or branched endovascular repair of complex aortic aneurysms. J Vasc Surg 69(2):357-366. https://doi.org/10.1016/j.jvs.2018. 05.216
3. Fairman AS, Wang GJ (2020) Spinal cord ischemia management: current indications and timing for drainage current indications for drainage, prophylactic versus selective drain protocols, and ideal timing if drainage is indicated

4. Poeck K, Hacke W (2006) Neurologie, 12. Aufl. Springer, Berlin, Heidelberg. ISBN3-540-29997-1

5. Ma JM, Tullius T, Van Ha TG (2019) Update on preoperative embolization of bone metastases. Semin intervent Radiol 36:241-248. https://doi. org/10.1055/s-0039-1693120

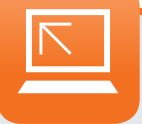

\section{Lesen Sie Gefässchirurgie online auf SpringerMedizin.de}

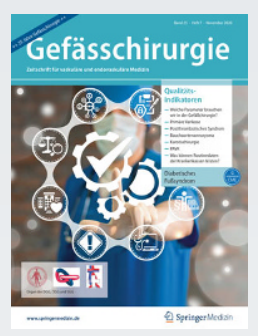

Auf SpringerMedizin.de erhalten Sie Zugang zu allen elektronisch verfügbaren Ausgaben und dem CME-Angebot Ihrer Zeitschrift - unabhängig davon, seit wann Sie Gefässchirurgie abonniert haben. Außerdem können Sie die Zeitschrift mit dem E-Paper auch bequem auf Ihrem Tablet lesen.

Alle Inhalte der Zeitschrift finden Sie unter www.springermedizin.de/gefaesschirurgie

> Genießen Sie die Vorteile der digitalen Nutzung:

- Greifen Sie auf alle Online-Inhalte der Zeitschrift zu - alle Jahrgänge, alle Ausgaben im Archiv.

- Laden Sie die kompletten ePaper-Ausgaben herunter.

- Lesen Sie OnlineFirst-Artikel vor der Druckveröffentlichung

- Profitieren Sie vom aktuellen CME-Angebot und frischen Sie Ihr CME-Punktekonto auf.

- Die Inhalte sind in verschiedenen Formaten verfügbar und können auf PC, Tablet oder Smartphone gelesen werden.

- Eine kluge Suchfunktion ermöglicht das Auffinden von Schlagworten innerhalb der Zeitschrift.

- Open-Access-Beiträge stehen Ihnen zeitschriftenübergreifend zur Verfügung.
So einfach erhalten Sie Zugang zum Online-Archiv:

- Registrieren Sie sich einmalig

auf www.springermedizin.de/register

- Geben Sie Ihre Einheitliche Fortbildungsnummer (EFN) an.

- Ihr Benutzername entspricht Ihrer E-Mail-Adresse, Ihr Passwort können Sie frei wählen und später jederzeit unter "Mein Profil" ändern.

- Geben Sie bei der Registierung die Lieferadresse Ihrer Zeitschrift an. Damit wird Ihr Abo-Zugang auf SpringerMedizin.de freigeschaltet.

Sind Sie bereits bei SpringerMedizin.de registriert? Dann wird Ihr Zeitschriftenabonnement automatisch Ihrem Online-Nutzerkonto hinzugefügt. Sollten die Angaben Ihres Online-Accounts nicht eindeutig mit den Angaben Ihres Zeitschriften-Abonnements übereinstimmen, kann die Zuordnung nicht sicher erfolgen. In diesem Fall und bei allen anderen Fragen zum Online-Zugang kontaktieren Sie bitte unseren Kundenservice.

Unser Kundenservice ist Ihnen bei Fragen und Problemen gerne behilflich:

Springer Customer Service Center GmbH

Kundenservice Zeitschriften

Tiergartenstr. 15, 69126 Heidelberg

Tel.: +49 $6221345-4303$

Fax: +496221 345-4229

E-Mail: leserservice@springer.com 